\title{
O uso da fotobiomodulação com luz LED e laser na clínica de ortodontia e ortopedia facial dos maxilares: uma revisão integrativa
}

The use of led light and laser photobiomodulation in the clinic of orthodontics and facial orthopedics of the jaws: an integrative review

El uso de luz led y fotobiomodulación láser en la clínica de ortodoncia y ortopedia facial de los maxilares: una revisión integradora

Recebido: 19/03/2021 | Revisado: 26/03/2021 | Aceito: 29/03/2021 | Publicado: 09/04/2021

\author{
Laís Gonzaga de Farias ${ }^{1}$ \\ ORCID: https://orcid.org/0000-0001-9362-7774 \\ Universidade Estadual da Paraíba, Brasil \\ E-mail: lais.gonzagaa@gmail.com \\ José de Alencar Fernandes Neto ${ }^{2}$ \\ ORCID: https://orcid.org/0000-0003-3711-6966 \\ Universidade Estadual da Paraíba, Brasil \\ E-mail: jneto411@hotmail.com \\ Maria Helena Chaves de Vasconcelos Catão ${ }^{3}$ \\ ORCID: https://orcid.org/0000-0001-7681-3225 \\ Universidade Estadual da Paraíba, Brasil \\ E-mail: mhelenact@zipmail.com.br
}

\begin{abstract}
Resumo
Os tratamentos ortodônticos ao longo dos últimos anos demandaram alternativas que pudessem deixá-los mais curtos e menos dolorosos. Estudos avaliaram que os lasers podem ser empregados nessa especialidade de forma satisfatória, como o laser de baixa potência que pode acelerar o tratamento ortodôntico, possuindo efeitos anti-inflamatórios, analgésicos e na resposta radicular. O objetivo da presente revisão integrativa foi revisar o uso da fotobiomodulação com luz led e laser dentro da especialidade da ortodontia e ortopedia facial. Como estratégia de busca, fez-se pesquisa bibliográfica nas bases de dados eletrônicas: PubMed, Lilacs e Web of Science, e selecionou-se estudos realizados em humanos e em laboratório in vivo com ratos, entre os anos 2010 e 2021 . Uma combinação das seguintes palavraschaves foi utilizada: luz LED, laser, in vivo, humanos e laboratório. Vinte e sete estudos foram selecionados, destes, treze foram eliminados como duplicatas e quatorze foram incluídos a fim de análise qualitativa. Em $64 \%$ dos artigos estudados, a terapia com o laser obteve resultados positivos, atuando de forma satisfatória aos objetivos propostos nos estudos, e os outros $36 \%$ não encontraram resultados satisfatórios. Após a análise dos estudos, pode-se concluir que a fotobiomodulação com laser de baixa intensidade e o LED são realmente eficazes, podendo ser utilizada como procedimento coadjuvante concomitante ao tratamento ortodôntico. Entretanto, há necessidade de mais conhecimento e estudos para que a terapia seja realmente incorporada ao dia a dia prático do clínico ortodontista.
\end{abstract}

Palavras-chave: Humanos; Laser; Ratos; Laboratório.

\begin{abstract}
Orthodontic treatments, over the last few years, required the development of shorter and less painful alternatives. Studies evaluated that lasers can be employed in this field satisfactorily. For example, low power laser therapy can accelerate orthodontic treatments, acting in algesia, showing anti-inflammatory, analgesic, and radicular response effects. This literature review aimed to review the application of photobiomodulation, LED light, and lasers in orthodontic and orthopedics facial. Our search strategy included bibliographic research performed in electronic databases, such as Pubmed, Lilacs, and Web of Science, and selected studies carried out in vivo and on human subjects between 2010 and 2021. Combinations of the following keywords were applied in our search: LED light, laser, in vivo, humans, and laboratory. The search resulted in twenty-seven studies. From those, thirteen were excluded as duplicates, and fourteen were included for qualitative analysis. In $64 \%$ of the papers included, laser therapy yielded positive results, satisfactorily achieving the studies' objectives. The remaining $36 \%$ did not attain satisfactory results. After analyzing the studies, it can be concluded that photobiomodulation with low-level laser is really effective and can be used as an adjunctive procedure concomitant with orthodontic treatment. However, there is
\end{abstract}

\footnotetext{
1 aMestranda em Odontologia, pelo Programa de Pós-Graduação em Odontologia da Universidade Estadual da Paraíba (UEPB), 58429-500, Campina Grande, Paraíba, Brasil.

2 bDoutorando em Odontologia, pelo Programa de Pós-Graduação em Odontologia da Universidade Estadual da Paraíba (UEPB), 58429-500, Campina Grande, Paraíba, Brasil.

3 cProfessora Doutora do Programa de Pós-Graduação em Odontologia da Universidade Estadual da Paraíba (UEPB), 58429-500, Campina Grande, Paraíba, Brasil. *e-mail: mhelenact@zipmail.com.br.
} 
a need for more knowledge and studies for the therapy to be really incorporated into the practical routine of the orthodontist clinician.

Keywords: Humans; Laser; Rats; Laboratory.

\begin{abstract}
Resumen
Los tratamientos de ortodoncia en los últimos años han demandado alternativas que pudieran hacerlos más, más cortos y menos dolorosos, estudios han evaluado que los láseres se pueden utilizar en esta especialidad de manera satisfactoria, como el láser de baja potencia que puede acelerar el tratamiento de ortodoncia, actuando en la algesia, teniendo efectos antiinflamatorios y analgésicos y en la respuesta radicular. El objetivo de esta revisión integradora fue revisar el uso de luz led y láser de fotobiomodulación dentro de la especialidad de ortodoncia y ortopedia facial. Como estrategia de búsqueda, se realizó una investigación bibliográfica en las bases de datos electronica's: PubMed, lilacs y web of science, y studios seleccionados realizados en el laboratorio in vivo y en humanos desde los años 2010 a 2021. Se combinó las siguientes palabras clave utilizado: luz led, láser, in vivo, humano y de laboratorio. se seleccionaron veinticinco estudios, de estos, trece se eliminaron como duplicados y doce se incluyeron para el análisis cualitativo. en el $64 \%$ de los artículos estudiados, la terapia con láser obtuvo resultados positivos, actuando satisfactoriamente a los objetivos propuestos en los estudios, el otro $36 \%$ no encontró resultados satisfactorios. tras analizar los estudios, se puede concluir que la fotobiomodulación con láser de bajo nivel es realmente eficaz y puede utilizarse como procedimiento coadyuvante concomitante al tratamiento de ortodoncia. sin embargo, se necesitan más conocimientos y estudios para que la terapia se incorpore realmente a la rutina práctica del ortodoncista.
\end{abstract}

Palabras clave: Humana; Láser; Ratones; Laboratorio.

\title{
1. Introdução
}

Desde o ano de 1960 os lasers foram incorporados na odontologia e sua aplicabilidade é principalmente na bioestimulação e cirurgia. O termo LASER é um acrônimo para Light Amplification by Stimulated Emission of Radiation, traduzindo-se como Amplificação da Luz por Emissão Estimulada de Radiação (Sant'ana, 2017).

Nas ciências da saúde, os lasers apresentam-se em grupos de acordo com a sua potência: laser de alta intensidade utilizado principalmente em ambientes cirúrgicos, devido a sua capacidade de corte de tecidos, e laser de baixa intensidade (LBI), comumente conhecido como laser terapêutico, com o objetivo de biomodulação a fim de ativação em processos regenerativos e cicatrizantes (Sousa, 2013).

Outra fototerapia reconhecida atualmente utiliza o LED (Light Emitting Diode), traduzindo-se como Diodo Emissor de Luz, caracterizado por diodos de semicondutores complexos e sólidos, que convertem corrente elétrica em um espectro luminoso estreito não coerente, em uma luz monocromática, conseguindo uma iluminação ampla (Chang et al., 2012).

A utilização do laser vem se tornando cada vez mais frequente na clínica odontológica nas mais diversas especialidades, como na Ortodontia, ciência que visa tratar maloclusões e anomalias nos dentes, mandíbula e face aplicando forças ortodônticas e obtendo uma ótima estética e função.

Os lasers na ortodontia podem ser utilizados de diversas formas, como na finalidade de: descolagem de bráquetes cerâmicos, polimerização da resina a fim de colagem de bráquetes e escaneamento intra-oral a laser, permitindo a captura de imagens tridimensionais da cavidade bucal dos pacientes (Graber, 2012). Nessa especialidade, a laserterapia de baixa intensidade é utilizada principalmente a fim de alívio na sintomatologia dolorosa, devido à remodelação óssea e movimento dentário proporcionado pelo aparelho ortodôntico. Sua eficácia é associada à frequência de ativações, a exemplo de expansões rápidas da maxila, acelerando a regeneração óssea, melhorando dessa forma a eficácia da movimentação (Cepera, 2012).

O laser de alta intensidade, vem se tornando bastante popular na prática ortodôntica, principalmente nos Estados Unidos, devido ao tratamento de forma rápida e eficaz das complicações dos tecidos moles associadas ao tratamento ortodôntico, melhorando a hemostasia, diminuindo o índice de infecções e reduzindo traumas e edemas (Graber, 2012).

Kravitz e Kusnoto (2012) destacam os benefícios da utilização da laserterapia na ortodontia: custo-benefício acessível, resposta agradável do paciente e a facilidade de uso, resultando em tratamentos mais eficazes e rápidos. Sabe-se também que essa terapia ativa a microcirculação e o metabolismo celular além de combater a dor em dois níveis: mediação de dor e 
estímulo na produção de endorfinas, englobando características altamente satisfatórias. Em busca de tais pontos positivos, o uso do laser dentro dessa especialidade vem se difundindo bastante na prática clínica dos profissionais.

As necessidades do ortodontista são muitas vezes específicas e únicas, portanto, a seleção do laser mais apropriado é fundamental e determinante. Pode-se citar alguns critérios que o clínico poderá elencar de acordo com os protocolos em questão: especificidade do procedimento, facilidade de uso, portabilidade e custo (Lomke, 2009).

A luz laser vem sendo empregada cada vez mais na odontologia, mostrando-se altamente eficaz quando bem empregada. Sabe-se que ela possui diversas indicações, podendo ser utilizada com efeitos de analgesia, corte e estimulação tecidual. Entretanto, o profissional deve entender e conhecer bem os tipos de lasers para que sua aplicabilidade seja a mais eficaz possível (Sant'anna et al., 2017).

Desta forma, o objetivo desta revisão integrativa foi analisar e revisar as indicações, possibilidades e desfechos de tratamentos utilizando o laser de baixa intensidade e o LED na ortodontia, permitindo uma visão mais clara ao clínico e especialista a fim de que tal terapêutica possa ser difundida e aplicada ao dia a dia prático, trazendo benefícios tanto para o profissional quanto para o paciente.

\section{Metodologia}

Este trabalho se trata de uma revisão de literatura integrativa qualitativa descritiva, pois contém informações relevantes na interpretação dos dados coletados, que contribuirão com os conhecimentos sobre este tema (Pereira et al., 2018). A intervenção analisada foi o uso do laser de baixa intensidade e o LED na especialidade da ortodontia e ortopedia facial, tomando-se como foco sua atuação e comparabilidade entre os grupos teste e os grupos controle.

Esta pesquisa foi conduzida de acordo com o seguinte critério de inclusão: ensaios clínicos controlados randomizados em humanos e in vivo em animais, nos idiomas português e inglês. Ensaios não controlados e monografias foram excluídos da presente revisão.

A estratégia de busca foi realizada por meio de uma pesquisa bibliográfica nas plataformas digitais e bases de dados eletrônicos PubMed, Lilacs e Web of Science realizada no mês de fevereiro de 2021, tomando-se como referência estudos relevantes publicados nos anos de 2010 a 2021. A seleção de artigos ocorreu mediante leitura sistemática e exploratória e a combinação das seguintes palavras-chave foram utilizadas: luz LED, laser, in vivo, humanos e laboratório.

\section{Resultados}

Foram identificados 27 estudos relacionados ao tema proposto. Após a exclusão de duplicatas, foram selecionados 14 artigos completos para revisão e análise qualitativa. Dos artigos revisados, quatro não obtiveram resultados favoráveis quanto à laserterapia, nos quais os grupos classificados como controle e tratamento, obtiveram respostas semelhantes, sem haver diferença potencialmente estatística entre eles. Os outros oito ensaios in vivo/clínicos, apresentaram respostas positivas ao tratamento a laser de baixa intensidade como na aceleração do movimento ortodôntico, alívio da dor após montagem do aparelho e ativação no metabolismo e remodelaçãoo óssea.

Dos 14 artigos científicos selecionados, foi avaliado o fator de impacto de cada um deles, que variaram de 0,8 a 4,678 com qualis capes classificados da seguinte forma: dois artigos A1, três artigos A2, dois artigos B2, três artigos B4, sendo quatro não classificados nesta plataforma. O processo de seleção e produção do estudo é mostrado através de um fluxograma ilustrativo demonstrado na Figura 1. 
Figura 1 - Fluxograma contendo o processo de busca dos estudos.

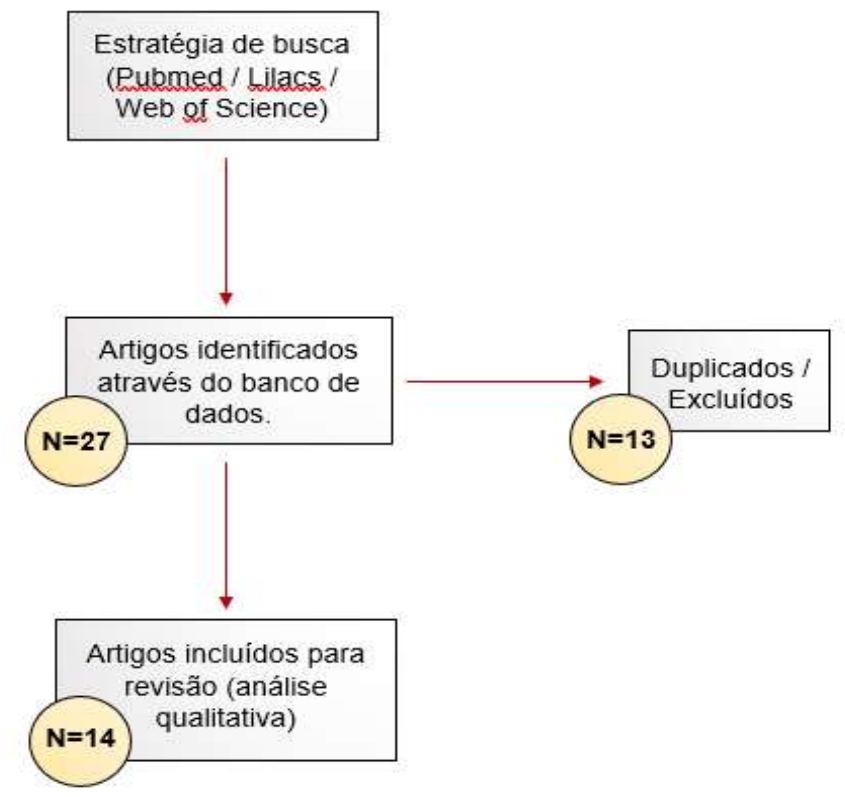

Fonte: Autores.

No Quadro 1 esposto a seguir, estão algumas informações como autores, ano de publicação, amostra, tipo de laser e principais resultados e conclusões dos artigos selecionados para essa revisão.

Quadro 1 - Informações dos artigos incluídos na revisão de literatura.

\begin{tabular}{|c|c|c|c|c|c|}
\hline AUTOR/ANO & DESENHO & $\begin{array}{l}\text { TAMANHO DA } \\
\text { AMOSTRA }\end{array}$ & $\begin{array}{l}\text { TIPO DE LASER } \\
\text { COMP. DE ONDA }(\mathbf{n m}) \\
\text { POTÊNCIA }(\mathbf{m W})\end{array}$ & $\begin{array}{c}\text { APLICAÇÃO/ DURAÇÃO/DOSE DE } \\
\text { ENERGIA }\end{array}$ & DESFECHO \\
\hline $\begin{array}{c}\text { Karabel et al. } \\
2021\end{array}$ & $\begin{array}{l}\text { Estudo in vivo } \\
\text { Ratos Wistar }\end{array}$ & $\mathrm{n}=30$ & $\begin{array}{l}\text { Diodo DECA } \\
980 \mathrm{~nm} \\
100 \mathrm{~mW}\end{array}$ & $\begin{array}{c}\text { Amostra dividida em três grupos, um } \\
\text { controle e dois experimentais }(\mathrm{n}=10 \mathrm{em} \\
\text { cada grupo. Irradiação por } 8 \text { dias, uma vez } \\
\text { por dia, com dois tempos de exposição nos } \\
\text { grupos teste: } \\
\mathrm{T} 1=12 \min (72 \mathrm{~J}) \\
\mathrm{T} 2=9 \min (54 \mathrm{~J})\end{array}$ & $\begin{array}{c}\text { Positivo } \\
\text { Aplicação do laser com Energia }=54 \mathrm{~J} \text { tem } \\
\text { se mostrada eficaz para acelerar o } \\
\text { movimento dentário ortodôntico. }\end{array}$ \\
\hline $\begin{array}{l}\text { Allazzawi et al. } \\
\qquad 2018\end{array}$ & $\begin{array}{l}\text { Estudo in vivo } \\
\text { Ratos Wistar }\end{array}$ & $\mathrm{n}=80$ & $\begin{array}{l}\text { GaAIAS/LIPUS } \\
\begin{array}{l}940 \mathrm{~nm} \\
30 \mathrm{~mW}\end{array}\end{array}$ & $\begin{array}{l}\text { Amostra dividida em quatro grupos. G1 } \\
\text { exposto ao laser de diodo; G2 exposto ao } \\
\text { laser LIPUS; G3 exposto aos dois tipos de } \\
\text { laser e G4 controle. } \\
\text { Irradiação em G1 por } 6 \text { minutos; G2 e G3 } \\
\text { por } 20 \text { minutos, todos uma vez ao dias, } \\
\text { durante sete dias. } \\
45 \mathrm{~J}\end{array}$ & $\begin{array}{c}\text { Positivo } \\
\text { A quantidade de movimento dentário e } \\
\text { remodelação óssea foi significativamente } \\
\text { maior nos grupos tratamento. }\end{array}$ \\
\hline $\begin{array}{c}\text { Gomes et al. } \\
2017\end{array}$ & $\begin{array}{l}\text { Estudo in vivo } \\
\text { Ratos Wistar }\end{array}$ & $\mathrm{n}=40$ & $\begin{array}{l}\text { GaAIAS } \\
780 \mathrm{~nm} \\
20 \mathrm{~mW}\end{array}$ & $\begin{array}{l}\text { Amostra dividida em oito grupos, com } \\
\text { irradição em tempos de } 10 \mathrm{~s}, 20 \mathrm{~s} \text { e } 40 \mathrm{~s} \text { uma } \\
\text { vez ao dia, em dias alternados durante } 21 \\
\text { dias em todos os grupos. Um total de } 10 \\
\text { doses foi aplicada em cada animal. } \\
\text { G1 (glicemia normal) } \\
\text { G2 (normoglicêmico, } 160 \mathrm{~J} \text { ) } \\
\text { G3 (normoglicêmico, } 320 \mathrm{~J} \text { ) } \\
\text { G4 (normoglicêmico, 640 J) } \\
\text { G5 (diabético) } \\
\text { G6 (diabético, 160 J) } \\
\text { G7 (diabético, 320 J) } \\
\text { G8 (diabético, 640 J) }\end{array}$ & $\begin{array}{c}\text { Negativo } \\
\text { Não houve efeito bioestimulador no } \\
\text { periodonto sob tratamento ortodôntico. }\end{array}$ \\
\hline
\end{tabular}


Research, Society and Development, v. 10, n. 4, e23110414084, 2021

(CC BY 4.0) | ISSN 2525-3409 | DOI: http://dx.doi.org/10.33448/rsd-v10i4.14084

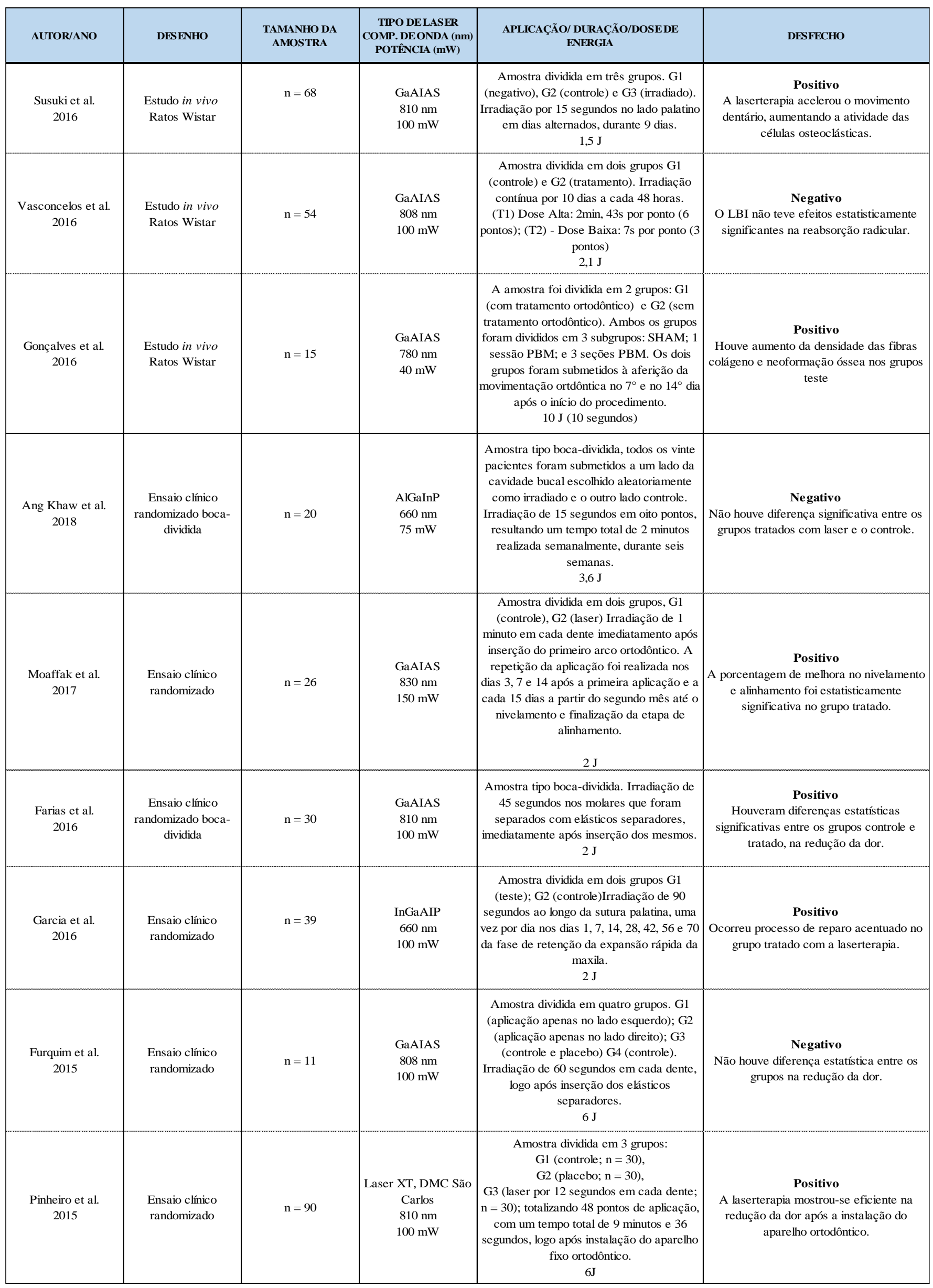




\begin{tabular}{|c|c|c|c|c|c|}
\hline AUTOR/ANO & DESENHO & $\begin{array}{c}\text { TAMANHO DA } \\
\text { AMOSTRA }\end{array}$ & $\begin{array}{c}\text { TIPO DE LASER } \\
\text { COMP. DE ONDA (nm) } \\
\text { POTÊNCIA (mW) }\end{array}$ & $\begin{array}{c}\text { APLICAÇÃO/ DURAÇãO/DOSE DE } \\
\text { ENERGIA }\end{array}$ & DESFECHO \\
\hline $\begin{array}{c}\text { Dalaie et al. } \\
2015\end{array}$ & $\begin{array}{c}\text { Ensaio clínico } \\
\text { randomizado boca- } \\
\text { dividida }\end{array}$ & $\mathrm{n}=12$ & $\begin{array}{c}\text { GaAlAs } \\
880 \mathrm{~nm} \\
100 \mathrm{~mW}\end{array}$ & $\begin{array}{c}\text { Amostra submetida à irradiação no lado } \\
\text { direito da maxila e o controle no lado } \\
\text { esquerdo } \\
5 \mathrm{~J}\end{array}$ & $\begin{array}{c}\text { Negativo radiação a laser mostrou-se ineficiente } \\
\text { quanto à moivemtnação dentária e melhora } \\
\text { na sintomatologia dolorosa }\end{array}$ \\
\hline $\begin{array}{c}\text { Qamruddin et al. } \\
2017\end{array}$ & $\begin{array}{c}\text { Ensaio clínico } \\
\text { randomizado } \\
\text { boca-dividida }\end{array}$ & $\mathrm{n}=22$ & $\begin{array}{c}\text { GaalAs } \\
980 \mathrm{~nm} \\
100 \mathrm{~mW}\end{array}$ & $\begin{array}{c}\text { Amostra tipo boca-dividida. Irradiação nos } \\
\text { dentes apinhados a cada três semanas, } \\
\text { durante } 10 \mathrm{~s} \\
2 \mathrm{~J}\end{array}$ \\
\hline
\end{tabular}

Fonte: Autores.

\section{Discussão}

Além de estudar a estética, a ortodontia se dedica ao conhecimento do complexo crânio facial, relacionando-se intimamente ao desenvolvimento da oclusão, tratamento de anomalias orofaciais e crescimento da face. Muitas são as nuances com as quais o ortodontista deve estar atento, a fim de se oferecer um melhor tipo de tratamento, como a saúde periodontal dos pacientes, fator preponderante para que haja uma adequada movimentação (Nobréga, 2013); e, higienização de bráquetes e fios ortodônticos que muitas vezes retém biofilme dental e podem retardar uma aceleração desejada (Kim et al., 2013).

A ortodontia contemporânea conta com várias técnicas auxiliares que visam principalmente um movimento dentário mais rápido e seguro, através de tecnologias nos fios, bráquetes e terapias complementares, como a utilização do laser. Muitos estudos correlacionam a ortodontia à terapia fotodinâmica, expondo suas utilidades e aplicações (Pol et al., 2016; Al Sayed, 2017). Vale ressaltar que a variedade de aparelhos e dosimetrias, geram dúvidas ao profissional que deseja utilizá-los, causando desinformação e uma certa subutilização de um recurso relevante para dia a dia clínico.

Muitas aplicações do laser de baixa intensidade são realizadas por meios ativos de diodo nos comprimentos de onda vermelhos $(632,660 \mathrm{~nm})$ e infravermelhos $(820,940 \mathrm{~nm})$. Os comprimentos de onda menores atingem uma camada de tecido menos profunda, assim como, os maiores atingem regiões mais profundas, como o infravermelho (Pamuk et al., 2017).

Estudos são realizados visando uma contribuição com a ciência a fim de que novas perspectivas sejam fomentadas, dessa forma, ensaios clínicos e in vivo são bastante propagados dentro da odontologia. A utilização de ratos geralmente é preferida pois foi relatado que a resposta biológica aos estímulos no osso periodontal e ligamento alveolar dos mesmos é semelhante à de humanos em nível celular, por tais razões ratos experimentais jovens são importantes em estudos que busquem reverberar resultados importantes para os seres humanos (Shirase et al., 2015).

Estudos experimentais realizados com animais verificaram efeitos favoráveis na aceleração e movimentação ortodôntica (Karabel et al., 2021; Susuki et al., 2016), havendo aumento na densidade das fibras colágeno (Gonçalves et al., 2016) e melhora no grau de remodelação óssea (Allazzawi et al., 2018), demonstrando uma continuidade de informações favoráveis.

Já em o estudo realizado por Vasconcelos et al., (2016) que avaliaram o grau de reabsorção radicular induzida pela movimentação ortodôntica em 54 ratos utilizando a laserterapia de baixa intensidade, não houve redução na reabsorção radicular inflamatória, não induzindo alteração na superfície radicular, assim como na conclusão de Gomes et al., (2017) que avaliaram o efeito bioestimulador do laser no periodonto de ratos diabéticos e não diabéticos, não havendo alteração estatisticamente significativa no tecido periodontal submetido ao tratamento ortodôntico.

Estudos clínicos randomizados como o realizado por Pinheiro (2015) que utilizou uma amostra de 90 indivíduos a fim de verificar o grau de algesia atingido pela laserterapia após a instalação do aparelho ortodôntico, verificou que houve considerável redução da dor, demonstrando um resultado positivo quanto à analgesia. 
Entretanto, Furquim et al., (2015) que buscaram encontrar resultados semelhantes a fim de melhoria na analgesia, só que neste caso, após a instalação de elásticos separadores pré bandagem ortodôntica, não encontraram significância estatística considerável, não havendo redução da dor nos pacientes submetidos à laserterapia, corroborando com Ang Khaw et al., (2018) em um estudo semelhante mas que objetivou avaliar o reparo de reabsorção radicular inflamatória induzido ortodonticamente, onde também nenhuma diferença significativa foi encontrada entre os grupos laser e controle.

Um ensaio randomizado de dois braços foi realizado por Moaffak (2017) utilizando uma amostra de 26 indivíduos com apinhamento dentário nos graus de moderado a severo nos incisivos centrais, que é conceituado pela falta de espaço para acomodação correta dos elementos; utilizou-se a terapia com o laser de baixa potência a fim de avaliar a possível aceleração na movimentação destes dentes. Após a submissão da amostra ao laser até a etapa final de alinhamento e nivelamento, que geralmente tem uma duração média de três meses, foi comprovada a efetividade da terapia, havendo uma aceleração considerável na movimentação dos dentes, diluindo o apinhamento de forma mais acelerada e menos dolorosa.

A dor é um dos principais efeitos negativos causados pelo tratamento ortodôntico. A aplicação de forças de tração e tensionamento sobre os dentes a fim de uma movimentação satisfatória são gatilhos para que possíveis desconfortos possam ocorrer (Huang, 2014). Dessa forma, alternativas como a laserterapia, foram utilizadas na especialidade da ortodontia, a fim de que a dor do paciente pudesse ser diminuída e, além da algesia, outros benefícios foram constatados através de estudos (Eslamian et al., 2013; Sousa et al., 2011) que relaram aceleração na movimentação ortodôntica utilizando-se o laser e ainda, melhora no grau de reabsorção radicular.

Long et al. (2015) realizaram uma meta-análise com o objetivo de avaliar e determinar a potência efetiva do laser de baixa intensidade na aceleração do movimento ortodôntico. Foram usadas as bases de dados Pubmed, Embase e Web of Knowledge abrangendo estudos dos anos de 1990 a 2013, avaliando-se cinco pesquisas tanto randomizadas, quanto não controladas. Uma heterogeneidade de resultados foi encontrada e uma possível explicação poderia ser os diferentes tipos de comprimento de onda utilizados, sendo estes: 650, 780, 800, e 860nm, havendo maior significância estatística no grupo que utilizou o comprimento de $780 \mathrm{~nm}$ e potência $20 \mathrm{~mW}$, já os demais comprimentos, densidades e potências obtiveram dados insuficientes para determinar uma melhor evidência.

Dalaie et al. (2015) realizaram um estudo utilizando a terapia com o laser de baixa intensidade em pacientes que iriam iniciar o tratamento ortodôntico, a fim de observar a possível algesia e também aceleração na movimentação. Um grupo de 22 pessoas foi utilizado, com uma idade média de 21,3 anos, os primeiros pré-molares superiores e inferiores foram extraídos, e foi feita a etapa inicial de alinhamento e nivelamento utilizando-se os fios 0.012 ', 0.014 ', 0.016 ', 0.018 ', 0.020 ” NiTi. Posteriormente, a etapa de retração de caninos foi realizada com fio 16x22 de aço, os indivíduos do grupo teste foram submetidos a irradiação com laser de baixa intensidade GaAlAs $\left(880 \mathrm{~nm}, 5 \mathrm{~J} / \mathrm{cm}^{2}\right)$ em oito pontos por dez segundos, em todas as faces de todos os dentes da arcada dentária, o nível de dor foi avaliado de acordo com uma escala confeccionada pelos autores. Não houve ao final do tratamento, diferenças significativas de movimentação dentária no grupo irradiado, assim como, não se observou diferença considerável no nível de dor desses pacientes, não havendo dessa forma, evidências de que o laser pode atuar dentro de tais perspectivas.

Miles (2017), examinou uma série de técnicas cirúrgicas e não cirúrgicas de aceleração na movimentação ortodôntica em uma revisão de literatura. As técnicas não cirúrgicas avaliadas foram o uso do laser de baixa intensidade, uso de medicação sistêmica a fim de alterar a resposta biológica, campos eletromagnéticos e correntes elétricas focais para modificar a biologia celular. Apenas a laserterapia dentre os métodos não cirúrgicos foi potencialmente importante na aceleração da movimentação. Dentre as técnicas cirúrgicas foram avaliadas: osteotomia, corticotomia e perfuração intra-óssea; a corticotomia mostrou-se eficiente quanto à aceleração na movimentação, mas a preferência por métodos menos invasivos, como a laserterapia de baixa intensidade, foi preferida pelos pacientes. 
Qamruddin et al. (2017), sucederam um estudo do tipo boca dividida em vinte e dois pacientes, a fim de se avaliar o grau de percepção da dor e também, a aceleração da movimentação ortodôntica. Vinte e dois indivíduos foram selecionados, todos eles apresentando maloclusão Classe II de Angle, havendo necessidade de extrações dos primeiros pré-molares superiores e inferiores. Após toda a etapa de alinhamento e nivelamento, que durou seis meses, iniciou-se a retração de caninos com arcos 0,019x0,025 de aço, molas fechadas NiTi de 6mm, com 150g de força aplicada. Dessa forma, a terapia com o laser foi iniciada, em todos os dentes nas faces vestibular e palatina, com cinco segundos de duração cada. O laser utilizado foi diodo de gálio com comprimento de onda de $980 \mathrm{~nm}$ e potência $100 \mathrm{~mW}$, a densidade de energia foi de $5 \mathrm{~J} / \mathrm{cm}^{2}$. A duração total desse estudo foi de quinze meses e os resultados foram altamente satisfatórios, os lados experimentais obtiveram uma resposta de movimentação ortodôntica duas vezes maior em relação ao lado controle, assim como, a dor era significativamente menor no lado experimental.

A divergência de resultados pode ser explicada pela diferença utilizada nos protocolos, tamanho da amostra, assim como, o tipo de laser, fato que ocorreu com alguns dos estudos inseridos na presente revisão (Furquim et al., 2015), (Garcia et al., 2016) (Pinheiro, 2015) que obtiveram amostras discrepantes e lasers de fabricantes distinto.

\section{Conclusão}

Após a análise dos estudos, pode-se concluir que a fotobiomodulação com laser de baixa intensidade e o LED são realmente eficazes, podendo ser utilizada como procedimento coadjuvante concomitante ao tratamento ortodôntico. Entretanto, há necessidade de estudos para que a terapia seja realmente incorporada ao dia a dia prático do clínico ortodontista. Sugerimos que novos trabalhos sejam realizados sobre esse tema, a fim de agregar conhecimentos para que o laser possa realmente ser utilizado dentro da clínica odontológica ortodôntica trazendo resultados comprovadamente eficazes aos tratamentos.

\section{Referências}

Alazzawi, M. M. J., Husein, A. \& Alam, M. K. (2018). Efeito do laser de baixa intensidade e da ultra sonografia pulsada de baixa intensidade na remodelação óssea durante a movimentação ortodontica em ratos. Rev. Prog Orthod, 19(10).

Alsayed Hasan, M. M. A., Sultan, K. \& Hamadah, O. (2017). Low-level laser therapy effectiveness in accelerating orthodontic tooth movement: A randomized controlled clinical trial. Angle, Orthodontist, 87(4), 499-504. Doi: 10.2319/ 062716-503.1.

Ang Khaw, C. M., Dalci, O., Foley, M., Petocz, P., Darendenliler, M. A \& Papadopoulou, A. K. (2018). Physical properties of root cementum: Part 27. Effect of low-level laser therapy on the repair of orthodontically induced inflammatory root resorption: A double-blind, split-mouth, randomized controlled clinical trial. American Journal of Orthodontics and Dentofacial Orthopedics, 3(154), 326 -336. Doi: 10.1016/ j.ajodo.2018.04.022.

Cepera, F., Torres, F.C., Scanavini, M. A \& Paranhos, L.R. (2012). Am J Orthod Dentofacial Orthop, 4(141), $444-50$.

Chang, C., Liu, S. S \& Roberts, W. E. (2012). Primary failure rate for 1680 extra-alveolar mandibular buccal shelf mini screws placed in movable mucosa or attached gingiva. The Angle Orthodontist, 85(6), 905-910. Doi.org/10.2319/092714.695.1.

Dalaie, K., Hamedi, R., Kharazifard., M. J., Mahdian M \& Bayat, M. (2015). Effect of low-level laser therapy on orthodontic tooth movement: a clinical investigation. J Dent (Tehran), 12(4), 249-256.

Eslamian, L., Farahani, A. B., Azhiri, A. H., Badiee, M. R \& Fekrazad, R. (2013). The effect of 810-nm low-level laser therapy on pain caused by orthodontic elastomeric separators. Lasers Med Sci, 29(2), 559-64.

Farias R. D., Closs, L. Q \& Miguens J. R., S. A. (2016). Evaluation of the use of low-level laser therapy in pain control in orthodontic patients: A randomized split-mouth clinical trial. Angle Orthodontist, 86(2), 193-198. Doi.org/10.2319/122214-933.1.

Furquim, R. D., Pascotto, R. C., Neto, J. R., Cardoso, J. R \& Ramos., A. L. (2015). Low-level laser therapy effects on pain perception related to the use of orthodontic elastomeric separators. Dental Press J. Orthod, 20(3). Doi.org/10.1590/2176-9451.20.3.037-042.oar.

Garcia, V.J., Arnabat, J., Comesana, R., Kasem, K., Ustrell J. M., Paseto, S., Segura., O. P., Cespedes, M. C. M \& Lobato., P.C. (2016). Effect of low-level laser therapy after rapid maxillary expansion: a clinical investigation. Lasers in Medical Science, 31(6), 1185-1194.

Gomes, M. F., Goulart, M. G. V., Gianasi, L. C., Hiraoka., C. M., Melo, G. F. S., Zangaro, R. A., Nóbrega, C. J. P \& Salgado, M. A.C. (2018). Effects of the photobiomodulation using different energy densities on the periodontal tissues under orthodontic force in rats with type 2 diabetes mellitus. Braz. Oral Res. Doi.org/10.1590/1807-3107bor-2018.vol32.0061. 
Gonçalves, C. F., Desiderá, A. C., Nascimento, G. C., Issa, J. P. M \& Panissi, C. R. A. L. (2016). Experimental tooth movement and phobiomodulation on bone remodeling in rats. Lasers Med Sci., 8(3) 264-267.

Graber L.W., Vanarsdall R.L \& Vig K.W.L. (2012). Ortodontia. Princípios e técnicas atuais. Rio de Janeiro: Elsevier.

Huang H., Williams R. C., \& Kyrkanies S. (2014). Movimento dentário ortodôntico acelerado: mecanismos moleculares. Am J Orthod Dentofacial Orthop.

Karabel M.A., Dogru, M., Dogru, A., Karadede., M. I \& Tuncer, M. C. (2020). Avaliação dos efeitos da aplicação do laser de diodo na movimentação dentária ortodôntica experimental em ratos. Análise histopatológica. Acta Cir. Bras, 12(2). Doi.org/10.1590/acb351204.

Kim,W. T., Bayome, M., Park. J. B., Park., J. H., Baek, S. H \& Kook H. A. (2013). Effect of frequent laser irradiation on orthodontic pain. A single-blind randomized clinical trial. Angle Orthod, 83(4), 611-6.

Kravitz, W.L.E.B \& Kusnoto, M. (2008). Lasers de tecidos moles em ortodontia: uma visão geral. Am J Orthod Dentofacial Orthop.

Lomke M.A. (2009). Clinical applications of dental lasers. Gen Dent, 57(1), 47-59.

Long, H., Zhou, Y., Xue, J., Liao, L., Ye, N., Whang, N \& Lai, W. (2015). The effectiveness of low level laser therapy in accelerating orthodontic tooth movement: a meta-analysis. Lasers $\mathrm{Med} S \mathrm{c}, 30(3), 1161-70$.

Miles, P. (2017). Accelerated Orthodontic Treatment-What's the evidence? Aust Dent J, 62(1), 63-70.

Moaffak, A.M., Hasan, A. A., Sultan, K \& Hamadah, O. (2017). Low-level laser therapy effectiveness in accelerating orthodontic tooth movement: A randomized controlled clinical trial. Angle Orthod , 87(4), 499-504. Doi.org/10.2319/062716-503.1.

Nóbrega C., Silva E. M \& Macedo C. R. (2013). Low-level laser therapy for treatment of pain associated with orthodontic elastomeric separator placement: a placebo-controlled randomized double-blind clinical trial. Photomed Laser Surg, 31(1), 10-6. Doi: 10.1089 / pho.2012.3338.

Pamuk, F., Lultifioglu, M., Aydogdu, A., Koyncunoglu C.Z., Cifcigasi, E \& Badur, O.S. (2017). The effect of low-level laser therapy as an adjunct to nonsurgical periodontal treatment on gingival crevicular fluid levels of transforming growth factor-beta 1, tissue plasminogen activator and plasminogen activator inhibitor 1 in smoking and non-smoking chronic periodontitis patients: A split-mouth, randomized control study. Journal of Periodontal Research, 52(5), 872882. Doi: $10.1111 /$ jre. 12457 .

Pereira, A. S., Shitsuka, D. M., Parreira, F. J. \& Shitsuka, R. (2018). Metodologia da pesquisa científica. Santa Maria, RS, Ed.UAB/NTE/UFSM.

Pinheiro, S. L., Agustinho, M. M. C., Martin, A. S \& Bueno, C. E. S. (2015). Efeito do laser de baixa potência na dor após a montagem do aparelho ortodôntico. Rev assoc paul cir dent, 69(4), 421-5.

Pol, R., Rugiero, T., Gallesio, G., Riso, M., Bergamasso, L., Mortellaro, C \& Mozzati, M. (2016). Efficacy of Anti-Inflammatory and Analgesic of Superpulsed Low Level Laser Therapy After Impacted Mandibular hird Molars Extractions. Journal of Craniofacial Surgery, 27(3), 685-690.

Qamruddin, I., Alam, M. K., Khamis, M. F \& HUSEIN, A. (2015). Minimally Invasive Techniques to Accelerate the Orthodontic Tooth Movement: A Systematic Review of Animal Studies. Biomed Res Int.

Sant'anna, E. F., Araújo, M. T. S., Nojima, L. I., Cunha, A. C., Silveira, B. L \& Marquesan, M. (2017). Aplicação do laser de alta intensidade em Ortodontia. Dente. Pressione J. Orthod, 22(2), 99. Doi: 10.1590 / 2177-6709.22.6.099-109.sar.

Shiraze., M., Sadegh M., Akhoundi A., Javadi E., Kamali A \& Motahhari P. (2015). Os efeitos do laser de diodo (660 nm) na taxa de movimentação dentária: um estudo animal. Lasers Med Sci, 30(2), 713-8. Doi.org/10.1590/1807-2577.14418.

Sousa, M. V. (2013). Avaliação do efeito do laser de baixa intensidade como recurso de ancoragem ortodôntica e na supressão da dor. Universidade de São Paulo, Rev. Faculdade de odontologia de bauru.

Sousa, M. V., Scavanini, M. A., Sanomya, E. K., Velasco, L. G \& Angelieri, F. (2011). Laser on the speed of orthodontic movement. Photomed Laser Surg, 29(3)191-6. Doi: 10.1089 / pho.2009.2652.

Suzuki, S. S., Garcez, A. S., Suzuki, H., Ervolino, E., Vencida, E \& Ribeiro, M. S. (2016). A terapia a laser de baixa intensidade estimula o metabolismo ósseo e inibe a reabsorção radicular durante a movimentação dentária em um modelo de roedor. J. Biophotonics, 9(11-12), 222-35. Doi.org/10.1002/jbio.201600016.

Vasconcelos, E. D., Henriques, J. F. C., Sousa, M. V. S., Oliveira, R. C., Consolaro, A., Pinzan, A., Henriques, F. P \& Bronfman, A. N. (2016). Ação do laser de baixo nível na reabsorção radicular induzida ortodonticamente: avaliação histológica e histomorfométrica. $J$ Lasers Med Sci, 7(3). 